\title{
Declining cardiovascular mortality and increasing obesity: a paradox
}

\section{Annika Rosengren MD}

$\infty \quad$ See related research paper by Lee and colleagues, page E55
$\mathrm{H}$ ow can the increased rates of obesity be reconciled with the rapid decrease in mortality related to coronary artery disease observed over the last decades in most Western countries? In the United States and Western Europe, rates of death from coronary artery disease declined almost linearly between 1970 and 2000. Modelling studies suggest that we can attribute $44 \%$ to $58 \%$ of the decline to population changes in risk factors, mainly cholesterol levels and smoking, and $36 \%$ to $47 \%$ to treatments. ${ }^{1-3}$ However, simultaneously, and seemingly at odds with these developments, is the increasing prevalence of obesity, ${ }^{4,5}$ which is a major risk factor for cardiovascular disease.

A worrying levelling off of the previously falling rates of death from coronary artery disease among young adults in England and Wales and the United States has recently been reported, even though the overall trends are still decreasing. ${ }^{6.7}$ This development could be in response to the obesity epidemic, with young people the most susceptible to this particular trend.

Another sign that all is not well is now being reported from Canada. In their analysis of data on risk factors from several national surveys from 1994 to 2005, Lee and colleagues $^{8}$ note that an estimated 1.29 million Canadians 12 years of age and older reported having heart disease in 2005. Compared with 1994, this represents an increase of $19 \%$ among males and 2\% among females, with an additional 380000 patients in 2005 compared with 1994. Although smoking declined in all age groups, self-reported obesity increased, more so among men than among women. For example, among men aged 35-49, obesity increased by $37 \%$, from $13.6 \%$ to $18.6 \%$. Among women, there was a barely detectable increase in the same age group, from $13.3 \%$ to 13.7\%. One in 5 Canadians aged 50-64 years reported being obese in 2005. This figure is still, however, below the figures reported from most states in the United States. Even so, the prevalence of self-reported hypertension and diabetes increased substantially among both men and women, and in nearly all age groups. Among men and women aged 65-74, the prevalence of self-reported hypertension rose by $52 \%$, from $32.9 \%$ to $50.1 \%$; for self-reported diabetes, the increase was by $32 \%$, from $11.2 \%$ to $14.7 \%$. Even though absolute rates of these risk factors were low among young people, the proportionate increases were greater.

Lee and colleagues also report important socio-economic differences. The age- and sex-adjusted prevalence of heart disease increased significantly among people in the lowest income category (by 27\%) and the low-mid income category (by 37\%).

\section{Key points}

- Increases in rates of obesity are seemingly at odds with simultaneous rapid decreases in rates of death from coronary artery disease.

- Although less smoking and better diet have contributed to declines in cardiovascular mortality, the obesity epidemic will likely fuel increases in diabetes and hypertension.

- Unless we address the emerging obesity epidemic, we risk seeing increased cardiovascular mortality in the future.

However, the prevalence rose by only $6 \%$ in the highest income group. Similarly, diabetes increased significantly in all income categories except the highest. The findings imply a widening gap for diabetes and heart disease between groups of different economic circumstances. However, although absolute rates of obesity remained higher in the low-income groups, a similar increase was observed in all income groups, which implied that the wealthy are not exempt from the effects of an obesogenic environment. In addition, obesity modified the rising burden of both diabetes and hypertension, with these conditions increasing the most among obese people.

What conclusions should be drawn? How can these opposing trends be reconciled within the same theoretical framework? More importantly, how can we explain decreasing rates of death from coronary artery disease observed simultaneously with increasing rates of obesity, given the strong correlation between obesity and several cardiovascular risk factors? A potential explanation is offered by recent findings from an analysis of 5 cross-sectional, nationally representative surveys in the United States ${ }^{9}$ that investigated the prevalence of high cholesterol, hypertension, current smoking and diabetes according to body mass index (BMI). The prevalence of all risk factors except diabetes decreased over time across all BMI groups; the greatest reductions were observed among overweight and obese people. Similarly, in a recent analysis of successive cohorts of 50-year-old Swedish men over a 40 -year period, ${ }^{10}$ serum cholesterol levels decreased to the same extent among normal weight, overweight and obese men. The mean serum cholesterol level among obese men in Sweden was $6.51 \mathrm{mmol} / \mathrm{L}$ in 1963 , as compared with $5.60 \mathrm{mmol} / \mathrm{L}$ in 2003 . This net decrease of $14 \%$ alone is enough to explain a substantial reduction in the rates of death

Annika Rosengren is with the Department of Emergency and Cardiovascular Medicine, Institute of Medicine, Sahlgrenska Academy, Göteborg, Sweden.

All editorial matter in CMAJ represents the opinions of the authors and not necessarily those of the Canadian Medical Association. 
from coronary artery disease. ${ }^{3}$ Accordingly, although obese people still have more risk factors (except for smoking) than lean people, the level of serum cholesterol is much lower than in previous decades. The main reason, thus, for the continuing decline in mortality associated with coronary artery disease seems to be driven by changes in diet, presumably the consumption of less saturated fat, and by less smoking. The obesity epidemic is driven by an obesogenic environment, with the marketing of and easy accessibility to calorie-dense foods, coupled with an environment that requires little or no physical exertion.

The rate of death from coronary artery disease has potentially reached its lowest point, or soon will - a development that will almost certainly display great geographic variation because of the multifaceted dimensions of cardiovascular disease. It remains to be seen whether the reported developments in heart disease and obesity-related risk factors in Canada, a country with obesity rates closer to those in Europe than in the United States, presage a levelling off of the decrease in cardiovascular mortality.

Political and educational actions seem to have been partly successful in decreasing cholesterol levels and rates of smoking. This inspires hope that fighting obesity could have at least some chance of succeeding, provided there is a collective understanding of the importance of emerging trends in the risk of cardiovascular disease. Clearly, this should also translate into evidence-based programs and population-based initiatives. All of this will require buy-in by political leaders and decision-makers in health systems worldwide. Whatever actions are taken, however, need to encompass society as a whole and not target only those at particularly high risk.

Competing interests: None declared.

\section{REFERENCES}

1. Unal B, Critchley JA, Capewell S. Explaining the decline in coronary heart disease mortality in England and Wales between 1981 and 2000. Circulation 2004;109:1101-7.

2. Ford ES, Ajani UA, Croft JB, et al. Explaining the decrease in US deaths from coronary disease, 1980-2000. N Engl J Med 2007;356:2388-98.

3. Björck L, Rosengren A, Bennett K, et al. Modelling the decreasing coronary heart disease mortality in Sweden between 1986 and 2002. Eur Heart J 2009;30:1046-56.

4. Ogden CL, Carroll MD, Curtin LR, et al. Prevalence of overweight and obesity in the United States, 1999-2004. JAMA 2006;295:1549-55.

5. James WP. The epidemiology of obesity: the size of the problem. J Intern Med 2008;263:336-52.

6. Ford ES, Capewell S. Coronary heart disease mortality among young adults in the U.S. from 1980 through 2002: concealed leveling of mortality rates. J Am Coll Cardiol 2007;50:2128-32.

7. O'Flaherty M, Ford E, Allender S, et al. Coronary heart disease trends in England and Wales from 1984 to 2004: concealed levelling of mortality rates among young adults. Heart 2007;94:178-81.

8. Lee DS, Chiu M, Manuel DG, et al. Trends in risk factors for cardiovascular disease in Canada: temporal, socio-demographic and geographic factors. CMAJ 2009. DOI:10.1503/cmaj.081629.

9. Gregg EW, Cheng YJ, Cadwell BL, et al. Secular trends in cardiovascular disease risk factors according to body mass index in US adults. JAMA 2005;293:1868-74.

10. Rosengren A, Eriksson H, Hansson PO, et al. Obesity and trends in cardiovascular risk factors over 40 years in Swedish men aged 50. J Intern Med 2009 Apr. 7. [Epub ahead of print].

Correspondence to: Dr. Annika Rosengren, Department of

Emergency and Cardiovascular Medicine, Institute of Medicine,

Sahlgrenska Academy, Göteborg SE-416 85, Sweden;

fax 4631 258933; annika.rosengren@gu.se
CANADIAN MEDICAL ASSOCIATION GENERAL COUNCIL 2009 SASKATOON, AUGUST 17-19

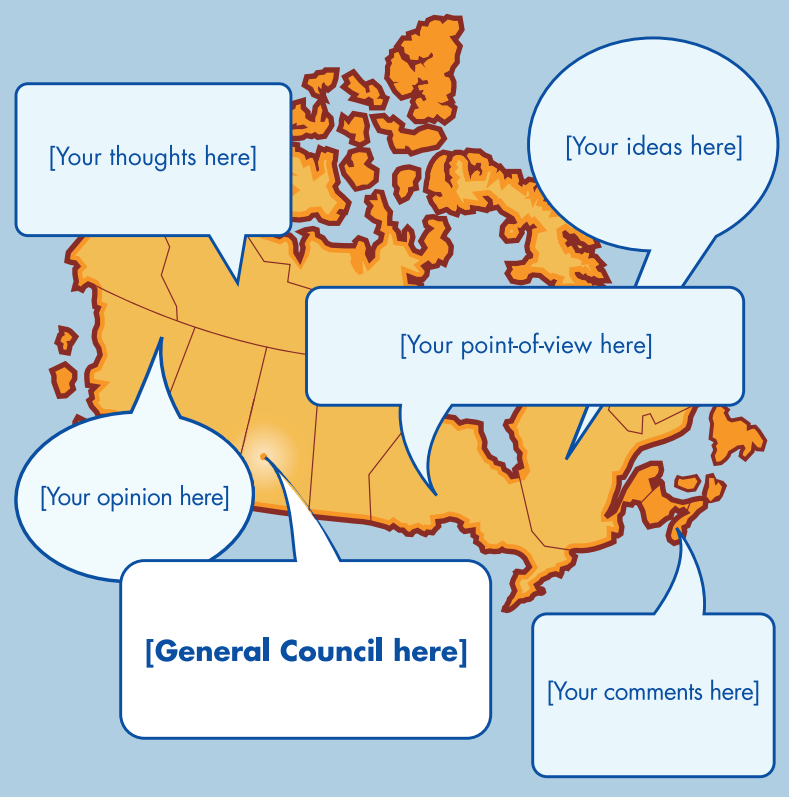

YOUR OPINIONS CAN MAKE IT TO SASKATOON EVEN IF YOU CAN'T.

CMA's General Council will be meeting from August $17^{\text {th }}$ to $19^{\text {th }}$ to make decisions that will guide our efforts in health care transformation in the coming year. To actively engage and involve CMA members, we're offering new participation opportunities, including:

- Members' Hour featuring live video feed and real-time engagement opportunities

- Online discussions at Asklepios, Canada's online physicians' community

\section{To discover the many ways you can participate, visit cma.ca/gc}

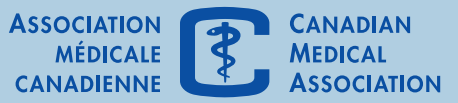

